\title{
BMJ Open Relationship between hospital performance measures and outcomes in patients with acute ischaemic stroke: a prospective cohort study
}

\author{
Xinmiao Zhang, ${ }^{1,2,3,4}$ Zixiao Li, ${ }^{1,2,3,4}$ Xingquan Zhao, ${ }^{1,2,3,4}$ Ying Xian, ${ }^{5}$ Liping Liu, ${ }^{1,2,3,4}$ \\ Chunxue Wang, ${ }^{1,2,3,4}$ Chunjuan Wang, ${ }^{1,2,3,4}$ Hao Li, ${ }^{1,2,3,4}$ Janet Prvu Bettger, ${ }^{5,6}$ \\ Qing Yang, ${ }^{6}$ David Wang, ${ }^{7}$ Yong Jiang, ${ }^{1,2,3,4}$ Xiaolei Bao, ${ }^{8}$ Xiaomeng Yang, ${ }^{1,2,3,4}$ \\ Yilong Wang, ${ }^{1,2,3,4}$ Yongjun Wang $1,2,3,4$
}

To cite: Zhang X, Li Z, Zhao X, et al. Relationship between hospital performance measures and outcomes in patients with acute ischaemic stroke: a prospective cohort study. BMJ Open 2018;8:e020467. doi:10.1136/ bmjopen-2017-020467

- Prepublication history for this paper is available online. To view these files, please visit the journal online (http://dx.doi. org/10.1136/bmjopen-2017020467).

$\mathrm{XZ}$ and ZL contributed equally. YW and YW contributed equally.

Received 13 November 2017 Revised 17 May 2018 Accepted 22 June 2018

Check for updates

(C) Author(s) (or their employer(s)) 2018. Re-use permitted under CC BY-NC. No commercial re-use. See rights and permissions. Published by BMJ.

For numbered affiliations see end of article.

Correspondence to Dr Yongjun Wang; yongjunwang1962@gmail.com

\section{ABSTRACT}

Objective Evidence-based performance measures have been increasingly used to evaluate hospital quality of stroke care, but their impact on stroke outcomes has not been verified. We aimed to evaluate the correlations between hospital performance measures and outcomes among patients with acute ischaemic stroke in a Chinese population. Methods Data were derived from a prospective cohort, which included 120 hospitals participating in the China National Stroke Registry between September 2007 and August 2008. Adherence to nine evidence-based performance measures was examined, and the composite score of hospital performance measures was calculated. The primary stroke outcomes were hospital-level, 30-day and 1-year risk-standardised mortality (RSM). Associations of individual performance measures and composite score with stroke outcomes were assessed using Spearman correlation coefficients.

Results One hundred and twenty hospitals that recruited 12027 patients with ischaemic stroke were included in our analysis. Among 12027 patients, 61.59\% were men, and the median age was 67 years. The overall composite score of performance measures was $63.3 \%$. The correlation coefficients between individual performance measures ranged widely from 0.01 to 0.66 . No association was observed between the composite score and 30-day RSM. The composite score was modestly associated with 1-year RSM (Spearman correlation coefficient, 0.34; $p<0.05$ ). The composite score explained only $2.53 \%$ and $10.18 \%$ of hospital-level variation in 30-day and 1-year RSM for patients with acute stroke.

Conclusions Adherence to evidence-based performance measures for acute ischaemic stroke was suboptimal in China. There were various correlations among hospital individual performance measures. The hospital performance measures had no correlations with 30-day RSM rate and modest correlations with 1-year RSM rate.

\section{INTRODUCTION}

Stroke remains to be the leading cause of mortality in China and was responsible for around 1.9 million deaths in $2013 .^{1}$ Although

\section{Strengths and limitations of this study}

- As the largest stroke database in China, hospitals participating in the China National Stroke Registry encompassed different regions and had good representativeness.

- We used the newer risk-standardised mortality method based on hierarchical models to account for variation in case numbers across hospitals or for intrahospital clustering effects.

- Our study was observational and non-randomised.

- The association between hospital performance measures and stroke outcomes did not necessarily prove causality.

evidence-based guidelines for stroke and transient ischaemic attack (TIA) have been developed and updated over time, ${ }^{2-4}$ there were variations and deficiencies in how these guidelines were applied. ${ }^{5-7}$ To reduce the national stroke burden, several national organisations have developed a set of evidence-based hospital performance measures to quantify and promote quality of stroke care..$^{7-9}$

Evidence-based hospital performance measures evaluated the structure, process and outcome of stroke care, and provided a metric that could be tracked, reported and improved. ${ }^{10}$ Evidence-based hospital performance measures have also been increasingly used for certification of stroke centres, ${ }^{11}$ public reporting ${ }^{12}$ and remuneration for performance. ${ }^{13}$ Although there has been substantial improvement in the quality of stroke care in China, gaps still exist in adherence to the evidence-based hospital performance measures. In addition, although measuring outcomes after stroke has important policy implications, ${ }^{14}$ there has been limited evidence demonstrating the 
association between adherence to performance measures and better patient outcomes. ${ }^{15}$

The China National Stroke Registry (CNSR) was a national, hospital-based, prospective stroke registry which encompassed different regions and had good representativeness. ${ }^{16}$ The CNSR thoroughly investigated all indicators related to the quality of stroke care, including evidence-based acute performance measures and performance measures at discharge. Thus, it could reflect the real situation of stroke care in China. Using data from the CNSR, we sought to determine whether evidence-based hospital performance measures were associated with short-term and long-term outcomes in patients with acute ischaemic stroke in China. Specifically, we (1) reported the hospital variations in adherence to evidence-based performance measures, (2) assessed the correlations between each individual performance measure, and (3) quantified the associations between the performance measures and hospital-level, risk-standardised, 30-day and 1-year mortalities among patients with acute ischaemic stroke in China.

\section{METHODS \\ CNSR overview}

The CNSR was the first nationwide, hospital-based, prospective stroke registry of quality assessment in China, conducted between September 2007 and August 2008. It was sponsored by the Ministry of Health of the People's Republic of China to establish a national stroke database to evaluate the quality of stroke care and outcomes. The study design of CNSR has been described previously. ${ }^{16}$ Briefly, the criteria used for hospital selection included the following: (1) having at least one stroke neurologist, (2) at least two sites were included from each of the 31 provinces and municipalities in mainland China, (3) commitment to participate voluntarily and (4) ability to conduct research. ${ }^{16} 17$ The CNSR recruited consecutive patients older than 18 years and diagnosed with ischaemic stroke, TIA, intracerebral haemorrhage or subarachnoid haemorrhage within 14 days of the index event. We collected patients' demographics, adherence to performance measures during hospitalisation, and status at discharge, 90-day, 6-month and 1-year follow-up after symptom onset. Written informed consent was acquired from the patient or the legally authorised representative.

\section{Data collection}

The characteristics of the hospitals were surveyed. According to the annual report on health statistics of China, ${ }^{18}$ hospitals were divided into three regions: eastern, central and western. Academic status was defined as being affiliated with a specific university or not. Hospital bed size was the number of total inpatient beds per site. Annual stroke discharge was the number of discharged patients diagnosed with stroke per year of each participating hospital.
Trained research coordinators at each hospital reviewed medical records daily to identify, obtain informed consent and enrol consecutive patients in accordance with the procedures indicated in a standard data collection manual. The key variables in the CNSR were assessed, including (1) demographic characteristics and medical history; (2) prestroke modified Rankin Scale (mRS) and National Institutes of Health Stroke Scale (NIHSS) scores on admission, vascular risk factors, and clinical care during hospitalisation; and (3) 30-day and 1-year all-cause mortalities after stroke. Patients or their authorised caregivers were contacted via telephone by trained research personnel with standardised scripts for follow-up at 90 days, 6 months and 1 year after stroke symptom onset. ${ }^{16}$ Specific death events and dates were recorded in detail, and 30-day and 1-year all-cause mortalities after stroke were confirmed.

\section{Performance measures}

We evaluated nine hospital performance measures, including seven 'core' measures for acute ischaemic stroke as recommended by the American Heart Association/American Stroke Association (AHA/ASA) Get With The Guidelines-Stroke (GWTG-Stroke) ${ }^{7}$ and two additional evidence-based secondary prevention metrics. ${ }^{4}$ These seven core performance measures included (1) intravenous tissue-type plasminogen activator in patients who arrived within 2 hours after symptom onset and treated within 3 hours (intravenous recombinant tissue-type plasminogen activator (rt-PA) <2 hours); (2) antithrombotic medication within 48 hours of admission (early antithrombotics); (3) deep vein thrombosis (DVT) prophylaxis within 48 hours of admission for non-ambulatory patients (DVT prophylaxis); (4) antithrombotic medication; (5) anticoagulation for atrial fibrillation (AF); (6) medications for lowering low-density lipoprotein (LDL) $\geq 100 \mathrm{mg} / \mathrm{dL}$; and (7) smoking cessation. The two additional performance measures were antihypertensive medication for patients with hypertension and hypoglycaemia medication for diabetes mellitus. Details of the performance measures are described in table 1.

Adherence to hospital-level individual performance measures was calculated as the proportion of patients who received the indicated care among all the patients who were eligible for the indicated care. The overall hospital performance measures were measured as the composite score, which was calculated by the total number of interventions actually performed among all eligible patients at a hospital, divided by the total number of recommended interventions in all eligible patients. ${ }^{19}$ Although the composite score was analysed as a continuous variable, hospitals were also divided, for descriptive purposes, into quartiles based on this continuous variable. To ensure the stability of the measure, hospitals with fewer than 20 patients in the denominator of any measures were excluded. 
Table 1 Specifications of evidence-based performance measures

\section{Performance measures of ischaemic stroke care

Acute performance measures
Intravenous rt-PA $<2$ hours

Early antithrombotics

DVT prophylaxis

\section{Definition of performance measures for eligible patients*}

Intravenous recombinant tissue plasminogen activator (rt-PA) in patients who arrived within 2 hours after initial symptom onset and treated within 3 hours.

\section{Antithrombotic therapy prescribed within 48 hours of hospitalisation, including antiplatelet} or anticoagulant therapy.

Patients at risk for deep vein thrombosis (DVT) (non-ambulatory) who received DVT prophylaxis by end of hospital day 2, including pneumatic compression, warfarin sodium or heparin sodium.

\begin{tabular}{ll} 
Performance measures at discharge \\
$\begin{array}{l}\text { Discharge antithrombotics } \\
\begin{array}{l}\text { Anticoagulation for atrial } \\
\text { fibrillation }\end{array}\end{array}$ & $\begin{array}{l}\text { Antithrombotic therapy prescribed at discharge. } \\
\text { documented during hospitalisation. }\end{array}$ \\
\hline LDL 100 & Lipid-lowering agent prescribed at discharge if low-density lipoprotein (LDL) $\geq 100 \mathrm{mg} / \mathrm{dL}$. \\
$\begin{array}{l}\text { Antihypertensive therapy for } \\
\text { hypertension }\end{array}$ & $\begin{array}{l}\text { Antihypertension medication prescribed at discharge for patients with a history of } \\
\text { hypertension or hypertension documented during hospitalisation. }\end{array}$ \\
$\begin{array}{l}\text { Hypoglycaemic therapy for } \\
\text { diabetes mellitus }\end{array}$ & $\begin{array}{l}\text { Hypoglycaemic medication prescribed at discharge for patients with a history of diabetes } \\
\text { mellitus or diabetes mellitus documented during hospitalisation. }\end{array}$ \\
\hline
\end{tabular}

*Eligible patients were those without any medical contraindications (eg, treatment intolerance, excessive risk of adverse reaction, patient/ family refusal or terminal illness/comfort care only) and documented as reasons for non-treatment for each of the applicable measures. We also excluded patients who were discharged to hospice, or another short-term general hospital, or against medical advice before the end of hospital day 2. For acute performance measures except for rt-PA measure, we excluded patients who died before the end of hospital day 2. For the acute rt-PA measure, we excluded patients with missing or erroneous onset, arrival or treatment times, those who began intravenous tissue-type plasminogen activator (t-PA) at an outside hospital, or who initiated intravenous t-PA after 180 min from onset. For performance measures at discharge, we excluded patients who died during hospitalisation. As for seven performance measures from the Get With The Guideline-Stroke (GWTG-Stroke), we employed the same criteria as the GWTG-Stroke.

\section{Risk-standardised mortality rates}

Risk-standardised mortality (RSM) rates were calculated using a multivariate hierarchical regression model in accordance with the AHA/ASA recommendations for risk adjustment of ischaemic stroke outcomes to compare hospital performance. ${ }^{15}$ The first level of the hierarchical model included patient characteristics. These patient variables were divided into three categories: (1) demographics and clinical features: age, gender, health insurance type (urban basic medical insurance schemes for urban and governmental employees and urban residents, new rural cooperative medical schemes for rural residents, commercial insurance, and self-payment), transport to hospital by emergency medical service and NIHSS score on admission; (2) vascular risk factors: hypertension, diabetes mellitus, dyslipidaemia, AF, coronary artery disease, previous myocardial infarction, congestive heart failure, valvular heart disease, history of stroke/TIA, peripheral vascular disease, current smoking and excessive alcohol consumption; and (3) other pre-existing comorbid conditions: chronic obstructive pulmonary disease, hepatic cirrhosis, peptic ulcer, previous gastrointestinal bleeding, Alzheimer's disease/ dementia, cancer, DVT/pulmonary embolus, renal dialysis, prestroke dependence $(\mathrm{mRS} \geq 3)$ and blood glucose on admission. The second level included hospital-specific random intercepts that allow for different baseline mortalities between hospitals. The hospital RSM rates were obtained as the ratio of 'predicted' to expected mortality, multiplied by the unadjusted mortalities for the total population. ${ }^{20} 21$ The expected outcome for each hospital was the number of deaths expected at the hospital if the hospital's patients were treated at a 'reference' hospital. The predicted hospital-level outcome was the number of expected mortalities at the 'specific' hospital and not at a reference hospital.

\section{Statistical analyses}

Numbers (percentages) were used to describe categorical variables, and median values with IQRs were reported for continuous variables. The correlations of individual performance measures, as well as performance measures and stroke outcomes, were evaluated using Spearman correlation coefficients. Bonferroni correction was performed in multiple comparisons. The interclass correlation (ICC) of 30-day and 1-year RSM rates was calculated. The relevant correlation coefficients and the percentage of the hospital-specific variations in RSM rates were explained using the squares of the correlation coefficients as indicators of the strength of the associations. ${ }^{22}$ 
All tests were two-tailed, and statistical significance was determined at the $\alpha$ level of 0.05 in univariate and multivariate analyses. Statistical analysis was performed using SAS V.9.3.

\section{Patient and public involvement}

No patients were involved in the planning of this study. Patients were recruited via the doctors and investigators at local hospitals in China. Once the results of the study have been published in a peer-reviewed journal, a summary of the main study findings will be distributed to the patients in Chinese language via the local doctors and investigators.

\section{RESULTS}

\section{Study hospitals and patient baseline characteristics}

Among 14526 eligible patients diagnosed with acute ischaemic stroke from 132 hospitals throughout China, complete 1-year follow-up information was available for 12173 patients (83.8\%). We excluded 12 hospitals with fewer than 20 patients in the denominator of any measures. Finally, 120 hospitals that recruited 12027 patients with ischaemic stroke were included in our analysis. Hospital and patient baseline characteristics are described in table 2. Among 120 hospitals, 62 (51.67\%) hospitals were teaching hospitals and $70(58.33 \%)$ were from eastern regions. The median hospital bed size was 1200 (IQR, 700-1861) and the annual stroke volume was 430 (IQR, 310-601). Among 12027 patients, 7407 $(61.59 \%)$ were men, and the median age was 67 (IQR, 57-75) years. Common vascular risk factors included hypertension, previous stroke/TIA, smoking history and diabetes. The median NIHSS score was 4 (IQR, 2-9).

\section{Variability in performance measures}

The overall composite score was $63.3 \%$ among the 120 hospitals. However, the composite score for each hospital varied considerably among the CNSR (table 3 ). The hospitals in the highest quartile (quartile 4) had a median (IQR) composite score of $85.1 \%$ (82.2\%-89.9\%), compared with $50.9 \%(44.2 \%-58.1 \%) \%)$ for those in the lowest quartile (quartile 1).

In table 3, we showed hospital rates (median and IQR) for each process measure, as well as 30-day and 1-year RSM rates. For acute interventions, the early antithrombotics showed the lowest degree of variance, although the difference between the first and third quartiles remained significant. In contrast, there were twofold to threefold differences in the use of intravenous rt-PA $<2$ hours, DVT prophylaxis, anticoagulant for $\mathrm{AF}$ and lipid-lowering medication for $\mathrm{LDL} \geq 100 \mathrm{mg} / \mathrm{dL}$.

\section{Correlations of performance measures}

The correlations between two individual hospital performance measures varied widely (table 4 ). After Bonferroni correction $(\mathrm{p}<0.001)$, we found moderate correlations (Spearman correlation coefficient $\geq 0.40 ; \mathrm{p}<0.001$ for all) for all pairwise comparisons between antithrombotic at discharge and lipid-lowering drugs for LDL $\geq 100 \mathrm{mg}$ / $\mathrm{dL}$, antihypertensive medication and antidiabetic medication, and between antidiabetic medication and antihypertensive medication and smoking cessation at discharge. Other hospital performance measures had modest correlations or no correlations with each other (Spearman correlation coefficient $<0.40$ for all).

\section{Association of performance measures with hospital-level RSM} rates

The median 30-day and 1-year RSM rates (IQR) were $5.41 \%(4.91 \%-6.17 \%) \%)$ and $13.55 \%(12.77 \%-14.48 \%)$, respectively (table 3 ). The ICC of 30-day RSM rate was 0.065 , and the ICC of 1-year RSM rate was 0.041.

All performance measures showed no correlations with 30-day RSM rate. Several performance measures showed statistically significant, but modest, correlations with 1-year RSM rate, including antithrombotics at discharge (Spearman correlation coefficient $=0.32$ ), antihypertensive medication (Spearman correlation coefficient $=0.30$ ) and antidiabetic medication (Spearman correlation coefficient $=0.31) \quad(\mathrm{p}<0.05$ for all $) \quad($ table 4$)$. These performance measures individually explained between $0.04 \%$ and $5.02 \%$ of hospital-level variation in 30-day RSM rate, and between $0.08 \%$ and $10.05 \%$ of hospital-level variation in 1-year RSM rate (table 5).

No correlation was observed between the hospital-level composite score and 30-day RSM rate (Spearman correlation coefficient $=0.16$ ). Modest correlation was observed between the hospital-level composite score and 1-year RSM rate (Spearman correlation coefficient $=0.32$ ). The composite score explained $2.53 \%$ and $10.18 \%$ of the hospital-level variation in 30-day and 1-year RSM rates, respectively (table 5 ).

\section{DISCUSSION}

The present study found that ischaemic stroke care was suboptimal in China and there were various correlations among hospital performance measures. The hospital-level composite score had no correlation with 30-day RSM rate and a modest correlation with 1-year RSM rate. This finding suggested hospital performance measures cannot reliably infer short-term and long-term mortalities after acute ischaemic stroke. Our results underscored that the current performance measures of stroke care provided complementary, but not redundant, information with the measures of 30-day and 1-year mortalities.

When comparing our data with the GWTG-Stroke in America, we found that ischaemic stroke care was suboptimal in China. The GWTG-Stroke programme was developed by the AHA/ASA as a national stroke registry and performance improvement programme with the primary goal of improving the quality of care and outcomes of stroke and TIA in America. ${ }^{714}$ The comparison showed that many hospital performance measures in China were far down from the GWTG-Stroke, ${ }^{14}{ }^{23}$ including 
Table 2 Baseline characteristics of patients with acute ischaemic stroke in the China National Stroke Registry

Level $\quad \mathrm{n}(\%)$

\section{Patient characteristics}

Total

12027

Demographics

$\begin{array}{llr}\text { Age, years } & \text { Median (IQR) } & 67(57-75) \\ \text { Gender } & \text { Male } & 7407(61.59) \\ \text { Insurance scheme } & \text { Female } & 4620(38.41) \\ & \text { UBMIS } & 7311(60.79) \\ & \text { NRCMS } & 2027(16.85) \\ & \text { Commercial } & 397(3.30) \\ \text { Transport to hospital by EMS } & \text { Self-payment } & 2292(19.06) \\ \text { NIHSS score on admission } & \text { Yes } & 1901(15.81) \\ & \text { Median (IQR) } & 4(2-9)\end{array}$

Vascular risk factors

Previous stroke/TIA

Yes

4088 (33.99)

Diabetes

Yes

$2593(21.56)$

Hypertension

Yes

7672 (63.79)

Dyslipidaemia

Yes

$1344(11.17)$

$\mathrm{CHD} /$ previous MI

Yes

$1748(14.53)$

Atrial fibrillation

Yes

$892(7.42)$

PVD

Yes

$76(0.63)$

Ever smoking

Yes

$4779(39.74)$

Heavy drinking

Yes

$1873(15.57)$

Pre-existing comorbid conditions

Congestive heart failure

Yes

$250(2.08)$

Valvular heart disease

Yes

$288(2.42)$

Chronic obstructive pulmonary disease

Yes

$138(1.15)$

Hepatic cirrhosis

Yes

$41(0.34)$

Peptic ulcer disease

Yes

$336(2.81)$

Previous gastrointestinal bleeding

Yes

$184(1.54)$

Dementia/Alzheimer's disease

Yes

$156(1.30)$

Cancer

Yes

$215(1.80)$

Deep venous thrombosis/pulmonary embolus

Yes

$77(0.64)$

Renal dialysis

Yes

$9(0.08)$

Prestroke dependence (mRS $>2$ )

Yes

$1120(9.31)$

Glucose on admission ( $\geq 7.5 \mathrm{mmol} / \mathrm{L})$

Yes

2328 (19.72)

Hospital characteristics

Total

120

Hospital size

Beds

Median (IQR)

$1200(700-1861)$

Hospital type

Teaching

Yes

$62(51.67)$

Annual stroke discharges

Median (IQR)

430 (310-601)

Geographical region

East

Yes

$70(58.33)$

Continued 
Table 2 Continued

\begin{tabular}{llc} 
& Level & $\mathbf{n}(\%)$ \\
\hline Middle & Yes & $26(21.67)$ \\
West & Yes & $24(20.0)$ \\
\hline
\end{tabular}

CHD, coronary artery disease; EMS, emergency medical service; MI, myocardial infarction; mRS, modified Rankin Scale; NIHSS, National Institutes of Health Stroke Scale; NRCMS, new rural cooperative medical scheme; PVD, peripheral vascular disease; TIA, transient ischaemic attack; UBMIS, urban basic medical insurance scheme.

the composite score $(63.3 \%$ in 2007 CNSR vs $94.0 \%$ in 2007 GWTG-Stroke), intravenous rt-PA $<2$ hours $(14.1 \%$ in 2007 CNSR vs $72.8 \%$ in 2007 GWTG-Stroke), early antithrombotics $(80.3 \%$ in 2007 CNSR vs $97.0 \%$ in 2007 GWTG-Stroke), DVT prophylaxis (59.6\% in 2007 CNSR vs $89.5 \%$ in 2007 GWTG-Stroke), discharge antithrombotics $(71.0 \%$ in 2012 CNSR vs $98.9 \%$ in 2007 GWTGStroke), anticoagulation for AF (19.7\% in 2007 CNSR vs $98.4 \%$ in 2007 GWTG-Stroke), lipid-lowering drug for $\mathrm{LDL} \geq 100 \mathrm{mg} / \mathrm{dL}$ ( $42.6 \%$ in 2012 CNSR vs $88.3 \%$ in 2007 GWTG-Stroke) and smoking cessation $(63.3 \%$ in 2007 CNSR vs $93.6 \%$ in 2007 GWTG-Stroke). Financial burden, lack of stroke centre certification and stroke education might be the main reasons.

The relationships between performance measures and stroke outcomes remained uncertain. Some studies have indicated that the use of performance measures was associated with lower short-term mortality and better functional outcomes. ${ }^{24-26}$ However, the links between these stroke performance measures and outcomes were not clear in other studies. ${ }^{27-30}$ There were several probable reasons for the modest correlations between hospital-level performance measures and RSMs in the present

\begin{tabular}{|c|c|}
\hline Measures & Median (IQR, \%) \\
\hline Composite score & $65.65(59.05-72.75)$ \\
\hline \multicolumn{2}{|l|}{ Acute interventions } \\
\hline Intravenous rt-PA $<2$ hours & $0.00(0.00-25.00)$ \\
\hline Early antithrombotics & 80.68 (73.21-88.24) \\
\hline DVT prophylaxis & $59.22(33.33-70.00)$ \\
\hline \multicolumn{2}{|l|}{ Discharge interventions } \\
\hline Discharge antithrombotics & $77.00(64.89-85.15)$ \\
\hline Anticoagulation for AF & 14.29 (0.00-26.97) \\
\hline LDL 100 & 41.89 (25.00-61.72) \\
\hline Antihypertensive medication & 61.37 (45.46-75.00) \\
\hline Antidiabetic medication & $67.03(53.15-79.58)$ \\
\hline Smoking cessation & 70.87 (50.55-83.33) \\
\hline \multicolumn{2}{|l|}{ RSM rate } \\
\hline 30-day & $5.41(4.91-6.17)$ \\
\hline 1-year & $13.55(12.77-14.48)$ \\
\hline
\end{tabular}

AF, atrial fibrillation; DVT, deep vein thrombosis; LDL, low-density lipoprotein; RSM, risk-standardised mortality; rt-PA, recombinant tissue-type plasminogen. study. First, although clinical trials have shown significant relationships between performance measures and outcomes, ${ }^{31}{ }^{32}$ the evidence-based performance measures were not mainly designed for overall short-term and longterm hospital mortalities. Second, there was relative little variation across hospitals in some performance measures, such as early antithrombotics after admission, which limited the ability to discriminate between hospitals based on their performance on these measures. Additionally, some performance measures, such as intravenous rt-PA, might have a greater effect regarding stroke-related disability than mortality. ${ }^{33}$ Finally, hospital mortalities, even risk-standardised ones, were more likely affected by many factors independent of the performance measures of stroke care quality, such as severity of stroke, patients' or their family's preference, economic level and clinical strategies that might contribute to a hospital's performance outcomes. ${ }^{34}$

The present study also demonstrated significant correlations between certain evidence-based performance measures for acute ischaemic stroke care, indicating that hospitals performing well in one performance measure were more likely to perform well in other measures. However, other performance measures were less strongly correlated with each other. This finding implied different performance measures reflected separate components of quality in acute ischaemic stroke care. Our work indicated that hospital performance rankings were likely to be substantially affected by the performance measures under selection. A broader range of performance measures may be needed to comprehensively reflect the quality of stroke care.

Our study had several strengths. First, to date, the CNSR is the largest stroke database in China. Hospitals participating in the CNSR encompassed different regions and had good representativeness. Second, we used the newer RSM method based on hierarchical models to account for variation in case numbers across hospitals or for intrahospital clustering effects. ${ }^{15}$ Third, the baseline characteristics of patients, such as age, gender, vascular risk factors, comorbid conditions and stroke severity (NIHSS score), were adjusted in calculating the RSMs in accordance with the AHA/ASA. ${ }^{15}{ }^{34}$ Additionally, besides short-term RSM, our study also analysed long-term RSM.

However, our study also had some limitations. First, our study was observational and non-randomised. The association between stroke care performance and stroke outcomes did not necessarily prove causality and may be 


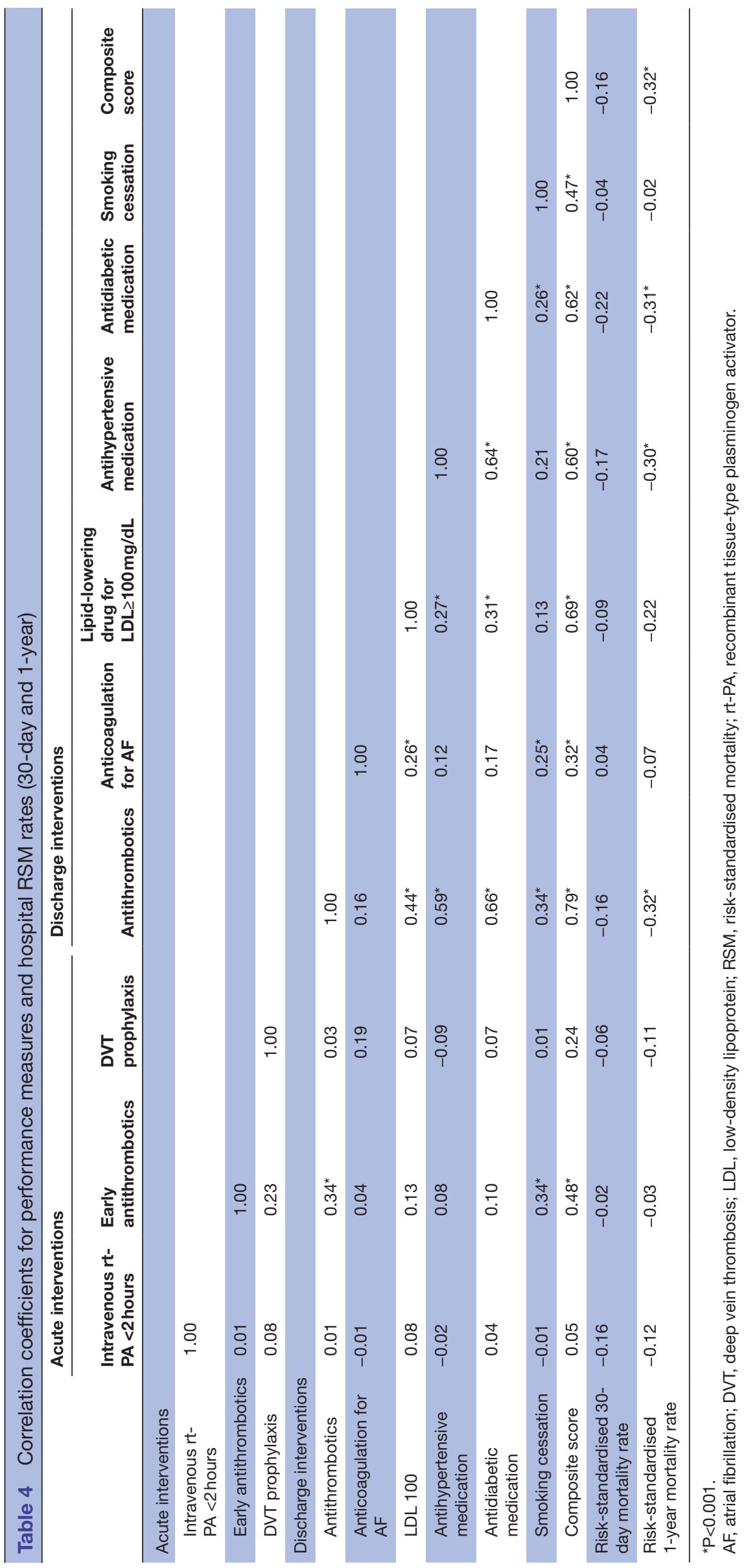

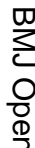

离

음

๙

is

$\stackrel{\circ}{\circ}$

ज़

을.

옹

N

今

윽

己

क्ष

옹

○

ํㅡㅇ

ญั

옥

吾

혹

금.

흠.

옥

음

을

กั

N్ల

एव

잉

궁

옴

음

एव

吕 
Table 5 Variance in 30-day and 1-year RSM rates explained by each performance measure and the composite measure

\begin{tabular}{|c|c|c|}
\hline & $\begin{array}{l}\text { 30-day RSM } \\
\text { rate }\end{array}$ & $\begin{array}{l}\text { 1-year RSM } \\
\text { rate }\end{array}$ \\
\hline Measures & $\begin{array}{l}\text { \% variance } \\
\text { explained }\end{array}$ & $\begin{array}{l}\% \text { variance } \\
\text { explained }\end{array}$ \\
\hline \multicolumn{3}{|l|}{ Acute interventions } \\
\hline Intravenous rt-PA $<2$ hours & 2.43 & 1.32 \\
\hline Early antithrombotics & 0.04 & 0.08 \\
\hline DVT prophylaxis & 0.38 & 1.14 \\
\hline \multicolumn{3}{|l|}{ Discharge interventions } \\
\hline Discharge antithrombotics & 2.59 & 10.05 \\
\hline Anticoagulation for $\mathrm{AF}$ & 0.14 & 0.45 \\
\hline LDL 100 & 0.76 & 4.67 \\
\hline Antihypertensive medication & 2.99 & 9.24 \\
\hline Antidiabetic medication & 5.02 & 9.49 \\
\hline Smoking cessation & 0.18 & 0.06 \\
\hline Composite score & 2.53 & 10.18 \\
\hline
\end{tabular}

AF, atrial fibrillation; DVT, deep vein thrombosis; LDL, low-density lipoprotein; RSM, risk-standardised mortality; rt-PA, recombinant tissue-type plasminogen activator.

confounded by previously discussed factors. Second, our outcome was mortality; other outcomes such as stroke recurrence and functional outcomes were also vital to patients, which should be assessed in future studies. ${ }^{30}$ Third, the hospital performance measures, stroke outcomes and the relationships between them might vary over time (such as extended time window for thrombolysis). Future research needs to determine the stability of the process-outcome relationship as quality improvement efforts would drive broader care adoption. Fourth, our data were collected between 2007 and 2008; therefore, interpretation of our data might be limited. Future research is needed to confirm our results. Finally, there were several differences in stroke healthcare delivery systems between China and other countries. The results reported herein came from the Chinese healthcare system and therefore may not necessarily be generalisable to other countries.

\section{CONCLUSIONS}

Adherence to evidence-based performance measures for acute ischaemic stroke was suboptimal in China. There were various correlations among hospital individual performance measures. The hospital performance measures had no correlations with 30-day RSM rate and modest correlations with 1-year RSM rate. More measures are needed to assess and improve the quality of hospital-level stroke care in China.
Author affiliations

${ }^{1}$ Department of Neurology, Beijing Tiantan Hospital, Capital Medical University, Beijing, China

${ }^{2}$ China National Clinical Research Center for Neurological Diseases, Beijing, China

${ }^{3}$ Center of Stroke, Beijing Institute for Brain Disorders, Beijing, China

${ }^{4}$ Beijing Key Laboratory of Translational Medicine for Cerebrovascular Disease, Beijing, China

${ }^{5}$ Department of Neurology, Duke Clinical Research Institute, Duke University, Durham, North Carolina, USA

${ }^{6}$ Duke University School of Nursing, Duke University, Durham, North Carolina, USA ${ }^{7}$ INI Stroke Network, OSF Healthcare System, University of Illinois College of Medicine, Peoria, Illinois, USA

${ }^{8}$ Statistical Analysis Office, Department of Information, General Hospital of Lanzhou Military Area Command, Lanzhou, Gansu, China

Acknowledgements We thank all participating hospitals, colleagues, nurses, imaging and laboratory technicians, and the CNSR steering committee members.

Contributors $\mathrm{XMZ}$ and $\mathrm{ZL}$ analysed the data and wrote the manuscript. $\mathrm{XB}, \mathrm{LL}$ CXW, HL, JPB, QY and DW analysed and interpreted the data. ZL, CJW, YJ, XY and XMZ performed the research. YJW, YLW, ZL, YX and XQZ conceived, designed and supervised the study.

Funding Supported by grants from the Ministry of Science and Technology of the People's Republic of China (2006BA101A11, 2008ZX09312-008, 2011BAI08B01, 2011BAI08B02, 2012ZX09303, 2013BAI09B14, 2013BAI09B03, 2015BAl12B02, 2015BAI12B04, 2017YFC1310900 and 2017YFC1310901), grants from the Beijing Municipal Science and Technology Commission (D15110700200000, D151100002015001, D151100002015002, Z161100000516223 and Z141107002514125), and the Beijing Tiantan Hospital Seeding Initiative.

Competing interests None declared.

Patient consent Not required.

Ethics approval Approval was obtained from the Central Institutional Review Board at Beijing Tiantan Hospital.

Provenance and peer review Not commissioned; externally peer reviewed. Data sharing statement № additional data are available.

Open access This is an open access article distributed in accordance with the Creative Commons Attribution Non Commercial (CC BY-NC 4.0) license, which permits others to distribute, remix, adapt, build upon this work non-commercially, and license their derivative works on different terms, provided the original work is properly cited, appropriate credit is given, any changes made indicated, and the use is non-commercial. See: http://creativecommons.org/licenses/by-nc/4.0/.

\section{REFERENCES}

1. Zhou M, Wang H, Zhu J, et al. Cause-specific mortality for 240 causes in China during 1990-2013: a systematic subnational analysis for the Global Burden of Disease Study 2013. Lancet 2016;387:251-72.

2. Sacco RL, Adams R, Albers G, et al. Guidelines for prevention of stroke in patients with ischemic stroke or transient ischemic attack: a statement for healthcare professionals from the American Heart Association/American Stroke Association Council on Stroke: co-sponsored by the Council on Cardiovascular Radiology and Intervention: the American Academy of Neurology affirms the value of this guideline. Stroke 2006;37:577-617.

3. Adams HP, del Zoppo G, Alberts MJ, et al. Guidelines for the early management of adults with ischemic stroke: a guideline from the American Heart Association/American Stroke Association Stroke Council, Clinical Cardiology Council, Cardiovascular Radiology and Intervention Council, and the Atherosclerotic Peripheral Vascular Disease and Quality of Care Outcomes in Research Interdisciplinary Working Groups: The American Academy of Neurology affirms the value of this guideline as an educational tool for neurologists. Circulation 2007:115:e478-e534.

4. Wang YJ, Zhang SM, Zhang L, et al. Chinese guidelines for the secondary prevention of ischemic stroke and transient ischemic attack 2010. CNS Neurosci Ther 2012;18:93-101.

5. Reeves MJ, Arora S, Broderick JP, et al. Acute stroke care in the US: results from 4 pilot prototypes of the Paul Coverdell National Acute Stroke Registry. Stroke 2005;36:1232-40. 
6. Hinchey JA, Shephard T, Tonn ST, et al. Benchmarks and determinants of adherence to stroke performance measures. Stroke 2008;39:1619-20.

7. Fonarow GC, Reeves MJ, Smith EE, et al. Characteristics, performance measures, and in-hospital outcomes of the first one million stroke and transient ischemic attack admissions in get with the guidelines-stroke. Circ Cardiovasc Qual Outcomes 2010;3:291-302.

8. LaBresh KA, Reeves MJ, Frankel MR, et al. Hospital treatment of patients with ischemic stroke or transient ischemic attack using the "Get With The Guidelines" program. Arch Intern Med 2008;168:411-7.

9. Ellrodt AG, Fonarow GC, Schwamm LH, et al. Synthesizing lessons learned from get with the guidelines: the value of diseasebased registries in improving quality and outcomes. Circulation 2013;128:2447-60.

10. Measuring and improving quality of care: a report from the American Heart Association/American College of Cardiology First Scientific Forum on Assessment of Healthcare Quality in Cardiovascular Disease and Stroke. Circulation 2000;101:1483-93.

11. Alberts MJ, Latchaw RE, Jagoda A, et al. Revised and updated recommendations for the establishment of primary stroke centers: a summary statement from the brain attack coalition. Stroke 2011;42:2651-65.

12. Krumholz HM, Keenan PS, Brush JE, et al. Standards for measures used for public reporting of efficiency in health care: a scientific statement from the American Heart Association Interdisciplinary Council on Quality of Care and Outcomes Research and the American College of Cardiology Foundation. Circulation 2008;118:1885-93.

13. Lindenauer PK, Remus D, Roman S, et al. Public reporting and pay for performance in hospital quality improvement. N Engl $J$ Med 2007;356:486-96.

14. Schwamm LH, Fonarow GC, Reeves MJ, et al. Get with the guidelines-stroke is associated with sustained improvement in care for patients hospitalized with acute stroke or transient ischemic attack. Circulation 2009;119:107-15.

15. Katzan IL, Spertus J, Bettger JP, et al. Risk adjustment of ischemic stroke outcomes for comparing hospital performance: a statement for healthcare professionals from the American Heart Association/ American Stroke Association. Stroke 2014;45:918-44.

16. Wang Y, Cui L, Ji X, et al. The China National Stroke Registry for patients with acute cerebrovascular events: design, rationale, and baseline patient characteristics. Int J Stroke 2011;6:355-61.

17. Wang $\mathrm{Y}$, Liao $\mathrm{X}$, Zhao $\mathrm{X}$, et al. Using recombinant tissue plasminogen activator to treat acute ischemic stroke in China: analysis of the results from the Chinese National Stroke Registry (CNSR). Stroke 2011;42:1658-64.

18. Ministry of health of the people's republic of china. Annual report on health statistics of china 2010 [in chinese]. Beijing, china: Publishing house of the peking union medical college, 2010.

19. Peterson ED, Delong ER, Masoudi FA, et al. ACCF/AHA 2010 Position Statement on Composite Measures for Healthcare Performance Assessment: a report of the American College of
Cardiology Foundation/American Heart Association Task Force on Performance Measures (Writing Committee to develop a position statement on composite measures). Circulation 2010;121:1780-91.

20. Shahian DM, Torchiana DF, Shemin RJ, et al. Massachusetts cardiac surgery report card: implications of statistical methodology. Ann Thorac Surg 2005;80:2106-13.

21. Krumholz HM, Wang Y, Mattera JA, et al. An administrative claims model suitable for profiling hospital performance based on 30-day mortality rates among patients with an acute myocardial infarction. Circulation 2006;113:1683-92.

22. Bradley EH, Herrin J, Elbel B, et al. Hospital quality for acute myocardial infarction: correlation among process measures and relationship with short-term mortality. JAMA 2006;296:72-8.

23. Li Z, Wang C, Zhao X, et al. Substantial Progress Yet Significant Opportunity for Improvement in Stroke Care in China. Stroke 2016;47:2843-9.

24. Tung YC, Jeng JS, Chang GM, et al. Processes and outcomes of ischemic stroke care: the influence of hospital level of care. Int J Qual Health Care 2015;27:260-6.

25. Hsieh FI, Lien LM, Chen ST, et al. Get With the Guidelines-Stroke performance indicators: surveillance of stroke care in the Taiwan Stroke Registry: Get With the Guidelines-Stroke in Taiwan. Circulation 2010:122:1116-23.

26. Micieli G, Cavallini A, Quaglini S, et al. Guideline compliance improves stroke outcome: a preliminary study in 4 districts in the Italian region of Lombardia. Stroke 2002;33:1341-7.

27. Adelman EE, Lisabeth LD, Smith MA, et al. Stroke Performance Measures Do Not Predict Functional Outcome. Neurohospitalist 2017;7:113-21.

28. McNaughton $\mathrm{H}, \mathrm{McPherson} \mathrm{K}$, Taylor $\mathrm{W}$, et al. Relationship between process and outcome in stroke care. Stroke 2003;34:713-7.

29. Parker C, Schwamm LH, Fonarow GC, et al. Stroke quality metrics: systematic reviews of the relationships to patient-centered outcomes and impact of public reporting. Stroke 2012;43:155-62.

30. Salinas J, Sprinkhuizen SM, Ackerson T, et al. An International Standard Set of Patient-Centered Outcome Measures After Stroke. Stroke 2016;47:180-6.

31. Jauch EC, Saver JL, Adams HP, et al. Guidelines for the early management of patients with acute ischemic stroke: a guideline for healthcare professionals from the American Heart Association/ American Stroke Association. Stroke 2013;44:870-947.

32. Kernan WN, Ovbiagele B, Black HR, et al. Guidelines for the prevention of stroke in patients with stroke and transient ischemic attack: a guideline for healthcare professionals from the American Heart Association/American Stroke Association. Stroke 2014;45:2160-236

33. Hacke W, Kaste M, Bluhmki E, et al. Thrombolysis with alteplase 3 to 4.5 hours after acute ischemic stroke. $N$ Engl $J$ Med 2008;359:1317-29.

34. Fonarow GC, Alberts MJ, Broderick JP, et al Stroke outcomes measures must be appropriately risk adjusted to ensure quality care of patients: a presidential advisory from the American Heart Association/American Stroke Association. Stroke 2014;45:1589-601. 